\title{
Postoperative analgesia for Enhanced recovery in Joint replacement: Audit of a new electronic prescribing order set
}

\author{
Jonathan Wright, Benjamin Cullinger, lan Bacarese-Hamilton
}

Whittington Hospital, London

\begin{abstract}
Enhanced recovery in joint replacement has been shown to reduce length of inpatient stay, reduce re-admission rates, and can improve early functional recovery. Postoperative analgesia is an important component of the group of interventions required to form a holistic enhanced recovery protocol. The introduction of electronic prescribing provides the opportunity to introduce some standardisation, where clinically appropriate, in the prescription of an evidence based postoperative analgesia protocol.

Enhanced recovery following joint replacement has been used at this institution since 2011. An order set for the postoperative analgesia protocol was introduced to the in house electronic prescribing system in August 2014 (JAC Medicines Management; JAC Computer Services Ltd., Basildon, UK). An audit was performed to follow the effect of the new system on compliance with the postoperative analgesia guidelines.

Improvements were seen following introduction of the electronic prescribing protocol in all criteria of the guideline with a demonstrated improvement in overall compliance from $0 \%$ to $35 \%$ in the first loop, with subsequent audit showing further improvement to $59 \%$ compliance.

Use of an embedded order set within an electronic prescribing system has demonstrated improved compliance with an enhanced recovery protocol. This ensures that the correct evidence based protocol is available to guide the junior clinician at the point of care, when the medication is being prescribed.
\end{abstract}

\section{Problem}

Postoperative pain is a complex symptom that requires a variety of different treatment modalities with the aim of enhancing efficacy of pain control whilst also minimising side effects (for example nausea, vomiting, constipation, and urinary retention). With this in mind, the Whittington Hospital (London, UK) pain team developed a multimodal analgesic protocol for patients undergoing elective total hip and knee replacements as part of the enhanced recovery programme.

Anecdotal evidence suggested that utilisation of this agreed protocol was poor, partly due to lack of awareness of the details of the protocol among those members of the team prescribing the analgesia. We set out to determine if this was indeed the case and if the utilisation of an electronic prescribing order set would enhance the uptake of a multimodal pain management protocol.

\section{Background}

Enhanced recovery after surgery is a concept involving a number of interventions aimed at reducing perioperative physiological stress and speeding the patient's recovery postoperatively. The use of preoperative education, modern anaesthetic techniques, multimodal pain management, and postoperative rehabilitation protocols each play a role in this technique. It has been used in several different surgical specialities demonstrating reduced length of stay[1].
Use of Enhanced recovery following total joint arthroplasty has been shown to result in improved patient satisfaction[2], reduced length of stay[3] without increased readmission rates[4], and reduced mortality rates[5].

Multimodal analgesia is aimed at effective pain control while minimising opiate requirements and reduced associated side effects, while allowing the patient to participate with physical rehabilitation [6]. The local protocol used at our centre has been produced over a period of time by consultant anaesthetists and members of the pain team.

\section{Baseline measurement}

For the baseline measurement in this quality improvement project data was collected prospectively for all arthroplasty patients who underwent surgery in a week at the beginning of July 2014 .

The in house electronic prescribing system was interrogated to assess the prescription of postoperative analgesia by doctors from the preoperative period through to discharge. Patient notes and blood results were reviewed to check for any reason for deviation from the standard protocol, according to the hospital guidelines (e.g. drug intolerances/allergies, use of non steroidal antiinflammatory drugs in patients with renal impairment). If no reason for changing the prescribed medications from the protocol was given, it was assumed that there was no valid clinical reason.

27 arthroplasty patients were included with no exclusions. This 
cycle demonstrated that the number of patients who received all medications according to the enhanced recovery protocol was $0 / 27$ in the baseline measurement. This clearly indicated that there was scope for improvement in compliance with the local postoperative analgesia protocol.

See supplementary file: ds5600.pdf - "Post operative analgesia protocol for enhanced recovery"

\section{Design}

JAC Medicines Management (JAC Computer Services Ltd., Basildon, UK) is an electronic prescribing interface that allows complex protocols to be prescribed as a simple order set. The system is well established in this hospital. As part of the prescribing options, certain pre-made "order sets" can be created to aid prescription of commonly combined medications (e.g. prescription of both oral and parenteral options of analgesia for perioperative patients).

The pharmacy department were able to incorporate the postoperative prescribing protocol for arthroplasty patients into an "order set" to assist the prescribing doctor in prescribing the correct medications, with the inclusion of planned "step down" and "breakthrough" analgesic options. The system allowed for modification of the protocol if the patient had a medical reason for one or more of the medications to be unsuitable.

Two order sets were introduced into the in house prescribing system in August 2014, one with the full protocol incorporated, the second with ibuprofen excluded for NSAID allergy patients.

\section{Strategy}

PDSA Cycle 1

All doctors receive training on use of the in house prescribing system as part of their trust induction. The use of a protocol as part of enhanced recovery for post operative analgesia in arthroplasty was explained to the junior doctors and the anaesthetic team members as part of their departmental induction. All had been familiarised with the use of the system and were confident with the use of simple order sets and were generally positive about their use. All members of the team were made aware of the introduction of the new order set as a streamlined means of prescribing the post operative analgesia protocol. The new order set was introduced in mid August 2014 with a plan to re-audit the compliance after 2 months.

\section{PDSA Cycle 2}

Following introduction of the intervention, the first audit cycle was completed and the encouraging results for this were presented as part of the regular orthopaedic team audit meeting. Generally the system was well received as straightforward to use. It was noted that while there was significant increase in the rate of compliance with the protocol, there still was some room for improvement. As there had been some rotation of junior staff within the department, it was evident that some of the new doctors were not fully familiar with the protocol. This was used as an opportunity to re-educate on the components of the protocol and the reasons for its use. The protocol, while available on the trust intranet, was also included as part of the induction documentation for the use by future cohorts. A further audit cycle was planned to monitor for ongoing compliance and assess any improvement following further education.

\section{Results}

A repeat of the data collection was performed initially in October 2014. This included all patients undergoing arthroplasty over the period of one month $(n=26)$ with the exclusion of one patient with chronic regional pain syndrome and sickle cell disease with complicated analgesic needs that the standard protocol could not effectively address. The total compliance with all parts of the protocol improved from $0 \%$ to $34.6 \%$ ( $p<0.001$, Fishers exact test). Greater improvements were seen with compliance to individual parts of the protocol: ibuprofen ( $4 \%$ pre intervention vs $58 \%$ post intervention), gabapentin (18\% vs $73 \%$ ), modified release morphine sulphate ( $44 \%$ vs $73 \%$ ), and weak opioid ( $26 \%$ vs $77 \%$ ) prescriptions all individually showed good improvements. Paracetamol was universally well prescribed $(100 \%$ in both measurements). Smaller improvements were made in the timing of conversion to PRN analgesia ( $89 \%$ to $96 \%$ ).

Following dissemination of the initial results and further education of the team the second data collection period was performed in February 2015. This continued to show improvement with the total compliance improving to $58 \%$. Similarly there were still improvements in parts of the protocol: gabapentin (96\%), modified release morphine sulphate $(96 \%)$, weak opioid $(100 \%)$, conversion to PRN analgesia (100\%). Despite these good improvements, the prescription of ibuprofen (58\%) according to protocol limited the number of patients in whom the prescription was totally compliant. It was noted that of these non protocol prescriptions 2/26 were prescribed incorrectly (wrong dose/timing) and 9/26 were excluded without documented contraindication.

See supplementary file: ds5599.pdf - "Chart demonstrating improvements in compliance following introduction of an electronic order set"

\section{Lessons and limitations}

Our results demonstrated a clear improvement in the rates of compliance with the postoperative analgesia protocol following introduction of the electronic order set. The use of order sets in an electronic prescribing system is relatively straightforward to learn and we believe that they are invaluable in situations where more complex medication regimes may be used, particularly when it is specific to a department (such as in rapid recovery following joint replacement) and a new member of the team may not have familiarity with such a protocol. This should be sustainable within the department and could be translatable to other specialties where suitable. 
A key lesson we found is that the change of junior staff within a department, without clear education at the time of induction can lead to incomplete uptake of a protocol. This was demonstrated by the further improvements we achieved following presentation of the initial results and re-education of the groups involved. The use of order sets forms part of the hospital induction and training on the prescribing system and education on the particular protocol is included in the departmental induction to ensure the improved compliance continues.

The numbers of this audit are not large, but reflect an average monthly turnover of elective hip and knee surgery patients within a district general hospital and the improvements are clear. There are limitations with the use of relatively short term data collection as they may miss data with team members away on leave or on night shifts.

We noted that while in the last cycle there were further good improvements, the total compliance was limited by the correct prescription of ibuprofen. It is not clear if this is due to a contraindication that was not recorded or evident from the notes, or if the protocol was not followed for another reason. With continued audit cycles to monitor compliance, this particular question could be investigated further.

As there is no additional cost in adding an order set to the electronic prescribing system this intervention is a cost effective way of improving compliance with a protocol.

\section{Conclusion}

Use of an electronic prescribing order set for postoperative analgesia in arthroplasty patients within an enhanced recovery programme demonstrates significantly improved compliance with the prescribing protocol. This is a technique which was generally well received, by the doctors using the system, as a means of easily utilising a relatively complicated protocol which may not be easily recalled. It may have a role for use within other hospitals or specialties where electronic prescribing is used.

\section{References}

1 Simpson JC, Moonesinghe SR, Grocott MP, Kuper M, McMeeking A, Oliver CM, Galsworthy MJ, Mythen MG; National Enhanced Recovery Partnership Advisory Board. Enhanced recovery from surgery in the UK: an audit of the enhanced recovery partnership programme 2009-2012. Br J Anaesth. 2015 Apr 29

2 Jones EL, Wainwright TW, Foster JD, Smith JR, Middleton RG, Francis NK. A systematic review of patient reported outcomes and patient experience in enhanced recovery after orthopaedic surgery. Ann R Coll Surg Engl. 2014 Mar;96(2):89-94.

3 Stambough JB, Nunley RM, Curry MC, Steger-May K, Clohisy JC. Rapid recovery protocols for primary total hip arthroplasty can safely reduce length of stay without increasing readmissions. J Arthroplasty. 2015 Apr;30(4):521-6.
4 Auyong DB, Allen CJ, Pahang JA, Clabeaux JJ, MacDonald KM, Hanson NA. Reduced Length of Hospitalization in Primary Total Knee Arthroplasty Patients Using an Updated Enhanced Recovery After Orthopedic Surgery (ERAS) Pathway. J Arthroplasty. 2015 May 12.

5 Malviya A, Martin K, Harper I, Muller S, Emmerson K, Partington $\mathrm{P}$, Reed M. Enhanced recovery program for hip and knee replacement. Acta Orthopaedica 2011; 82 (5): 577-581 577

6 Lamplot JD, Wagner ER, Manning DW. Multimodal pain management in total knee arthroplasty: a prospective randomized controlled trial. J Arthroplasty. 2014 Feb;29(2):329-34.

\section{Declaration of interests}

Nothing to declare

\section{Acknowledgements}

The Whittington hospital pharmacy team, with particular thanks to Rajesh Babu (JAC pharmacist), and the Whittington hospital pain team.

\section{Ethical approval}

This project was performed following approval from the local audit and clinical governance department. 